\title{
Author Correction: DSYB catalyses the key step of dimethylsulfoniopropionate biosynthesis in many phytoplankton
}

Andrew R. J. Curson, Beth T. Williams, Benjamin J. Pinchbeck, Leanne P. Sims, Ana Bermejo Martínez, Peter Paolo L. Rivera, Deepak Kumaresan, Elena Mercadé (D), Lewis G. Spurgin, Ornella Carrión, Simon Moxon, Rose Ann Cattolico, Unnikrishnan Kuzhiumparambil, Paul Guagliardo, Peta L. Clode (D), Jean-Baptiste Raina and Jonathan D. Todd (D)

Correction to: Nature Microbiology https://doi.org/10.1038/s41564-018-0119-5, published online 26 February 2018.

In the version of this Letter originally published, the Methods incorrectly stated that all phytoplankton cultures were sampled in midexponential phase. The low-nitrogen cultures were sampled in early stationary phase and at the point at which Fv/Fm values decreased, to indicate that cultures were experiencing low-nitrogen conditions. All other phytoplankton cultures were sampled in exponential phase. Growth and Fv/Fm data are provided here on high- and low-nitrogen cultures (Figs. 1, 2 and 3) to clarify and support this correction. The Methods also stated that cell counting was done using a Beckman Multisizer 3 Coulter Counter, but a CASY Model TT Cell Counter was used.

In addition, in Supplementary Fig. 2e,f, the $x$-axis tick-mark label "High salinity (50 PSU)" should have read "High salinity (70 PSU)" in both panels.

The authors apologize for any confusion they may have caused, and acknowledge T. Mock and J. Strauss for raising these discrepancies. The errors have now been corrected. 

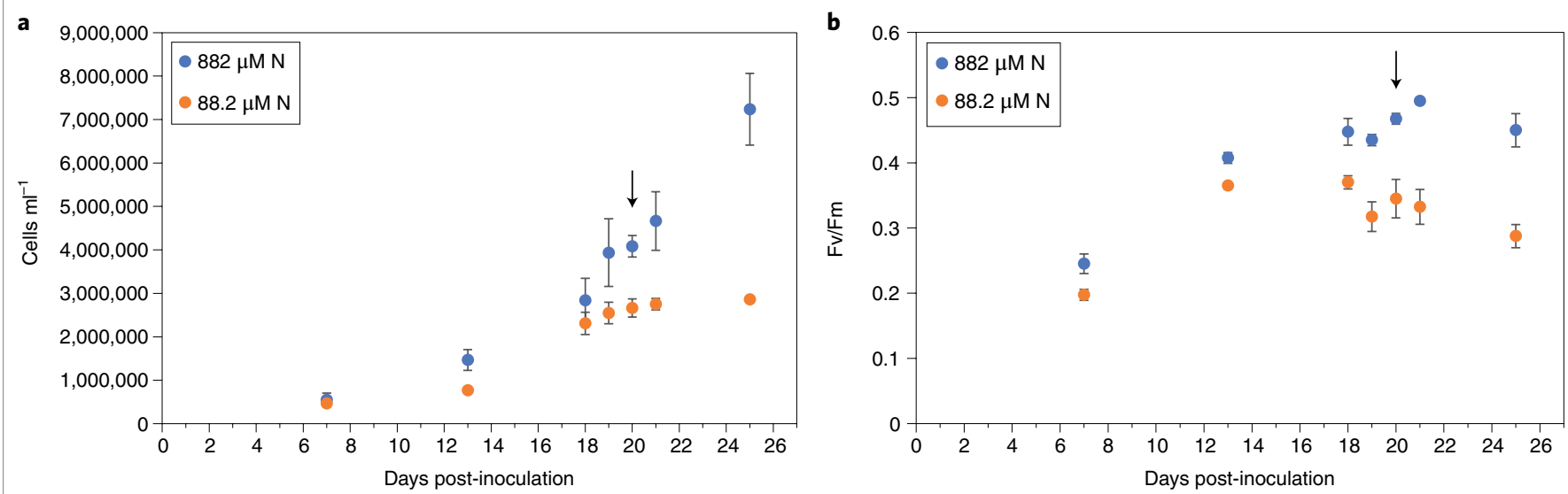

Fig. 1 | Fragilariopsis cylindrus growth data for high and low nitrogen cultures. $\mathbf{a}$, Cells $\mathrm{ml}^{-1}$. b, Fv/Fm. Average of replicates $(n=4)$ with error bars showing standard deviation. Arrow indicates sampling time for all cultures.
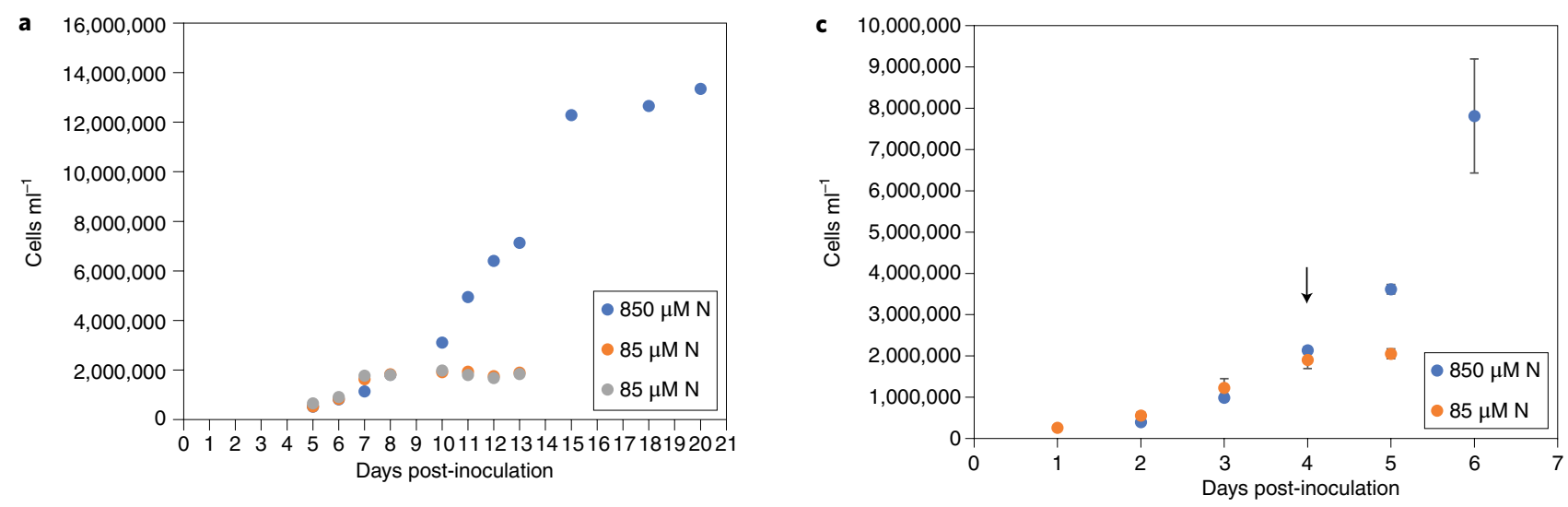

b

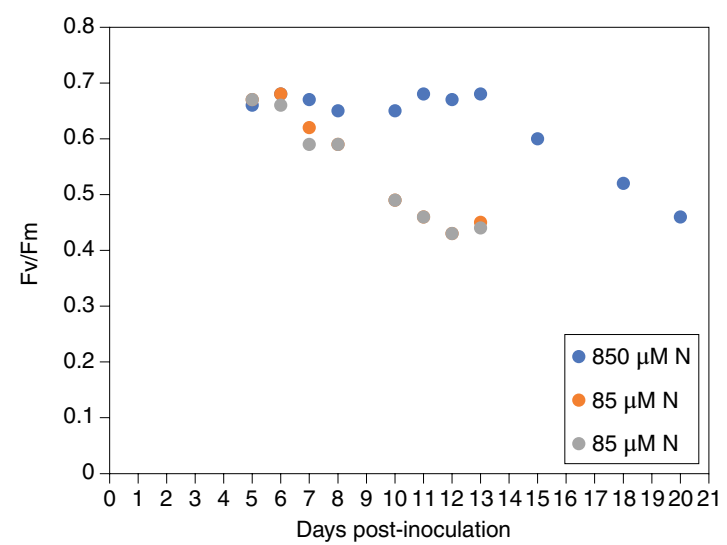

d

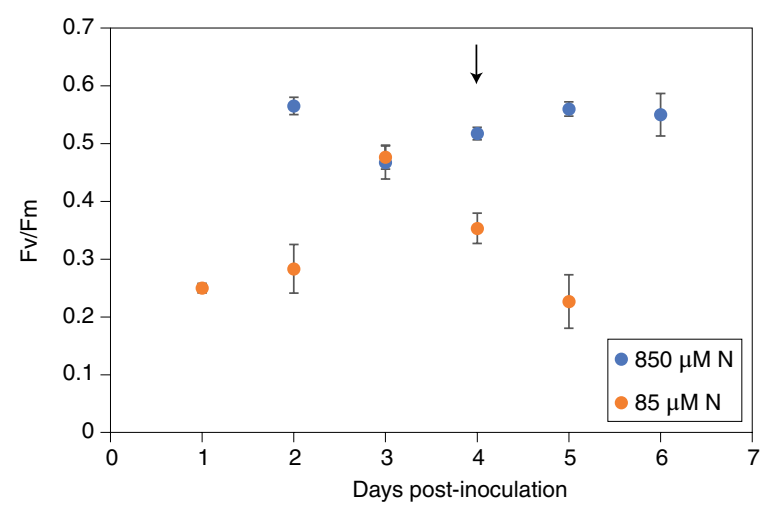

Fig. 2 | Chrysochromulina tobin growth data for high and low nitrogen cultures. a, Preliminary growth test cells $\mathrm{ml}^{-1}$. $\mathbf{b}$, Preliminary growth test Fv/Fm. c, Final experiment cells $\mathrm{ml}^{-1}$. d, Final experiment Fv/Fm. Each dataset (differentiated by colour) represents a single replicate for preliminary experiment in $\mathbf{a}$ and $\mathbf{b}$. Panels $\mathbf{c}$ and $\mathbf{d}$ show average of replicates for standard nitrogen $(n=4)$ and low nitrogen $(n=3)$ with error bars representing standard deviation. Arrows indicate sampling times for all cultures. 


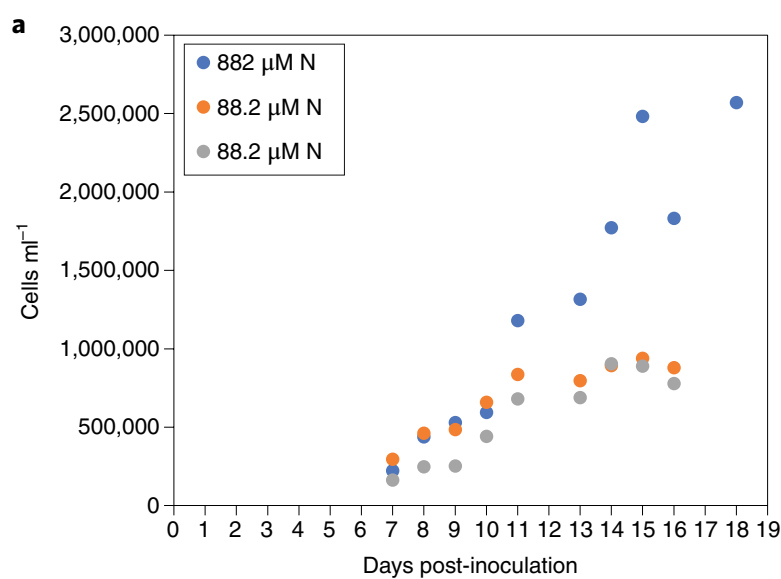

b

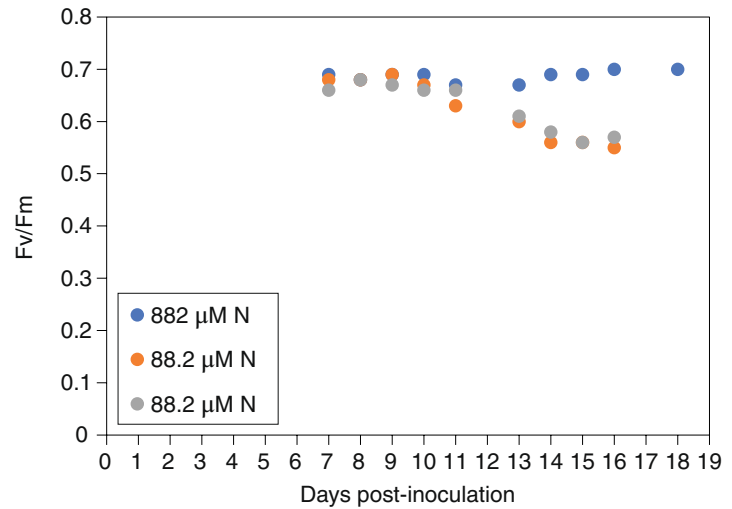

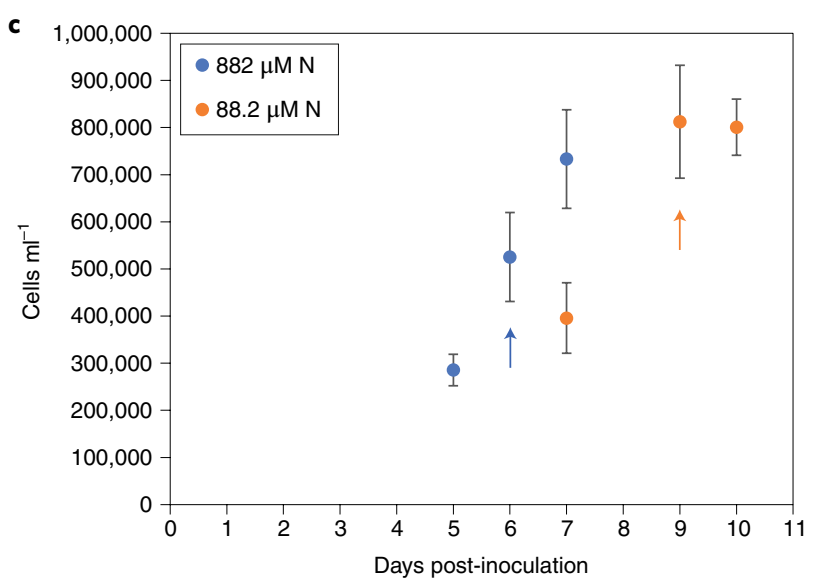

d

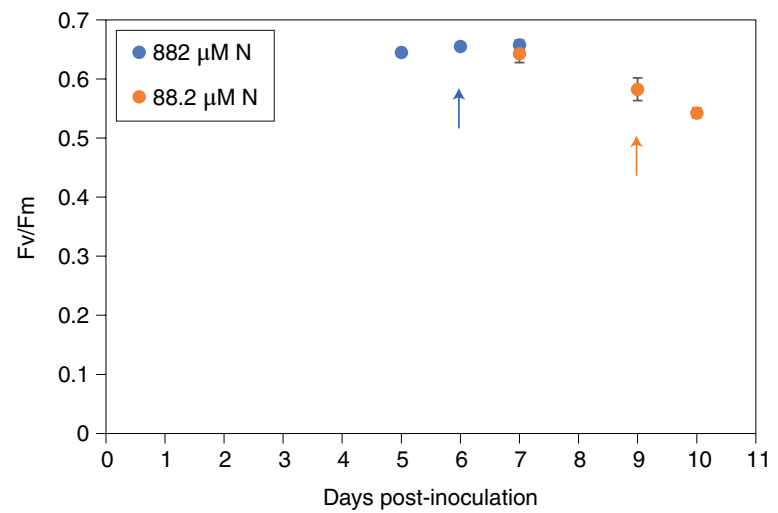

Fig. 3 | Prymnesium parvum growth data for high and low nitrogen cultures. a, Preliminary growth test cells $\mathrm{ml}^{-1}$. b, Preliminary growth test Fv/Fm. $\mathbf{c}$, Final experiment cells $\mathrm{ml}^{-1}$. d, Final experiment Fv/Fm. Each dataset (differentiated by colour) represents a single replicate for preliminary experiment in $\mathbf{a}$ and $\mathbf{b}$. Panels $\mathbf{c}$ and $\mathbf{d}$ show average of replicates $(n=4)$ with error bars representing standard deviation. Arrows indicate sampling times (colourcoded according to sample type).

Published online: 30 January 2019

https://doi.org/10.1038/s41564-019-0386-9 